
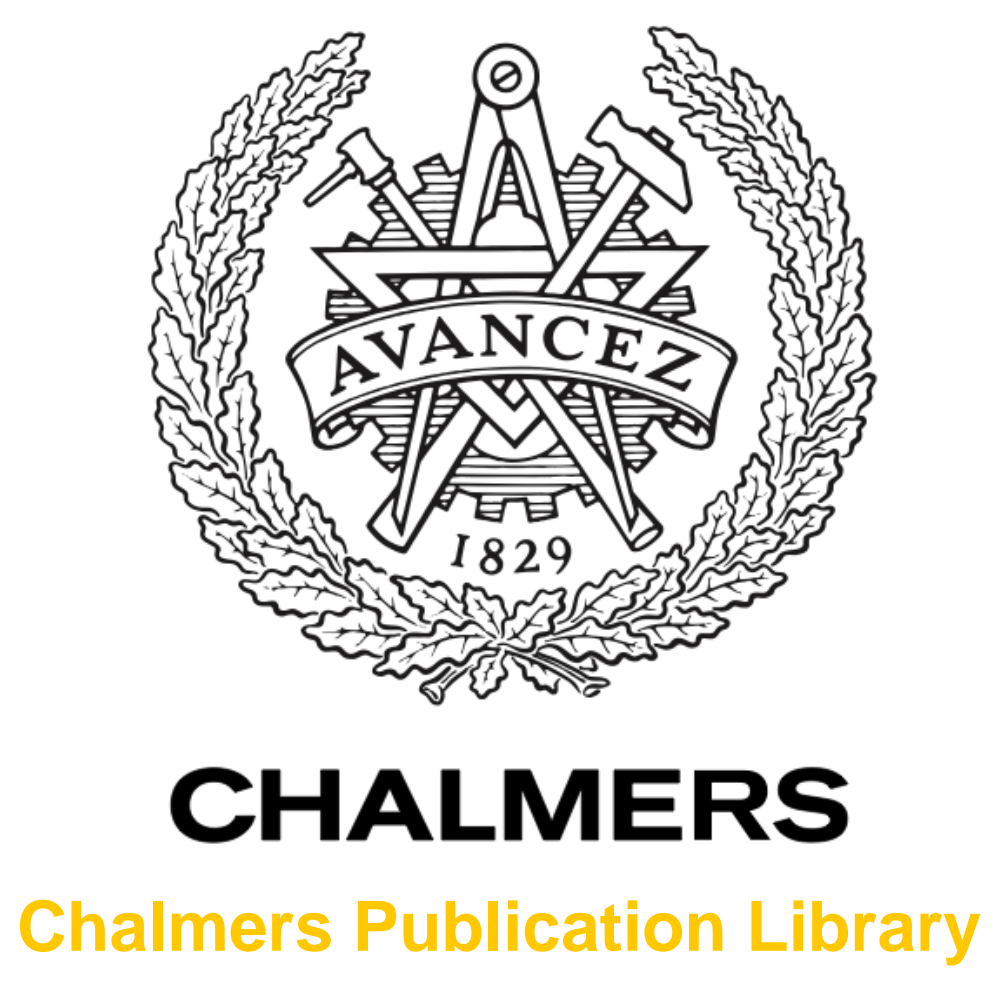

\title{
Power Optimization in Nonlinear Flexible-Grid Optical Networks
}

This document has been downloaded from Chalmers Publication Library (CPL). It is the author's version of a work that was accepted for publication in:

\section{European conference on optical communication}

Citation for the published paper:

Agrell, E. ; Wymeersch, H. ; Li, Y. et al. (2015) "Power Optimization in Nonlinear FlexibleGrid Optical Networks". European conference on optical communication

Downloaded from: http://publications.lib.chalmers.se/publication/223989

Notice: Changes introduced as a result of publishing processes such as copy-editing and formatting may not be reflected in this document. For a definitive version of this work, please refer to the published source. Please note that access to the published version might require a subscription. 


\title{
Power Optimization in Nonlinear Flexible-Grid Optical Networks
}

\author{
Li Yan, Juzi Zhao, Erik Agrell, and Henk Wymeersch
}

Chalmers University of Technology, Gothenburg, SE-41296, Sweden. (lyaa@chalmers.se)

\begin{abstract}
The routing and spectrum assignment in nonlinear flexible-grid networks is extended by optimizing the power separately for each connection. This yields $24 \%$ bandwidth reduction for large networks. An Optimization formulation and decomposition strategy are proposed to solve the problem.
\end{abstract}

\section{Introduction}

Flexible-grid optical networks are a promising solution to the exponentially increasing Internet traffic with different modulation formats, bandwidths, carrier frequencies, or even power spectral densities (PSDs) coexisting. In such networks, nonlinear impairments due to both inter- and intrachannel interference become significant. Accurate channel models are available that account for nonlinear interference $(\mathrm{NLI})^{1,2}$. Based on these models, routing and spectrum allocation algorithms in flexible optical orthogonal frequency division multiplexing (OOFDM) networks have been demonstrated advantageous in bandwidth savings $^{3}$ with equal PSD for all connections. Nonlinear channel models have also been used in fixedgrid networks ${ }^{4}$ to optimize the traffic throughput or bandwidth usage with variable connection PSDs. However, the optimization of PSD per connection has only been studied for individual flexible-grid links ${ }^{5,6}$, not for entire networks.

In this paper, we address the problem of resource allocation with variable individual connection PSD for offline requests in transparent flexible-grid networks with OOFDM utilizing a nonlinear $\mathrm{NLI}$ model ${ }^{1}$. The maximum bandwidth usage is minimized while all connections' SNR requirements are satisfied.

\section{Nonlinear Model and Linear Approximation}

We use the Gaussian noise (GN) model $^{1}$ to estimate the NLI and signal-to-noise ratio (SNR). The NLI PSD for connection $i \in \mathcal{D}$ is written as $G_{i}^{\mathrm{NLI}}=$ $G_{i}^{\mathrm{SCl}}+G_{i}^{\mathrm{XCl}}$, where $G_{i}^{\mathrm{SCl}}=3 \gamma^{2} N_{i}^{\mathrm{s}} F_{i i}^{2} G_{i}^{3} / \alpha^{2}$ and $G_{i}^{\mathrm{XCl}}=\sum_{j \in \mathcal{D}, j \neq i} 6 \gamma^{2} N_{i j}^{\mathrm{s}} F_{i j}^{2} G_{i} G_{j}^{2} / \alpha^{2}$ are the selfchannel interference (SCl) and cross-channel interference $(\mathrm{XCl})$, respectively, $N_{i}^{\mathrm{s}}$ is the number of spans transversed by connection $i, N_{i j}^{\mathrm{s}}$ is the number of spans shared by $i$ and $j, \mathcal{D}$ is the set of all connections, $G_{k}$ is the PSD of connection $k, \gamma$ is the fiber nonlinear parameter, $\alpha$ is the fiber power attenuation. $F_{i j}^{2}$ represents the approximate NLI effect, which is expressed

The research was funded by the Swedish Research Council (VR) under grant no. 2012-5280. as $F_{i j}^{2}=2 / \xi\left(\operatorname{Im}\left[\operatorname{Li}\left(\Delta f_{i} / 2\left(\Delta f_{i} / 2+f_{i j}\right)\right)\right]+\right.$ $\left.\operatorname{Im}\left[\operatorname{Li}\left(\Delta f_{i} / 2\left(\Delta f_{i} / 2-f_{i j}\right)\right)\right]\right)$, where Im is imaginary part of a complex number, $\mathrm{Li}(\cdot)$ is the dilog function, $\xi=4 \pi^{2}\left|\beta_{2}\right| / \alpha, \beta_{2}$ is group velocity dispersion, $\Delta f_{i}$ is the bandwidth of connection $i$, and $f_{i j}$ is the channel spacing between connections $i$ and $j$. Signals also suffer from amplified spontaneous emission (ASE) noise, with PSD as $G_{i}^{\mathrm{ASE}}=\left(e^{\alpha L}-1\right) h \nu n_{\mathrm{sp}}$, where $L$ is fiber span length, $n_{\mathrm{sp}}$ is the spontaneous emission factor, $\nu$ is the light frequency, and $h$ is Planck's constant.

The SNR for connection $i$ is $\mathrm{SNR}_{i}=$ $G_{i} /\left(G_{i}^{\mathrm{ASE}}+G_{i}^{\mathrm{NLI}}\right)$, and its modulation format $m_{i}$ is chosen from $\mathcal{M}=\{$ PM-BPSK, PM-QPSK, PM8QAM, PM-16QAM $\}$, the SNR requirement $\mathrm{SNR}_{i} \geq \mathrm{SNR}_{m_{i}}^{\mathrm{th}}$ should be met, or equivalently $G_{i}^{\mathrm{ASE}} / G_{i}+G_{i}^{\mathrm{SCl}} / G_{i}+G_{i}^{\mathrm{XCl}} / G_{i}-1 / \mathrm{SNR}_{m_{i}}^{\mathrm{th}} \leq 0$.

Since $G_{i}^{\mathrm{ASE}} / G_{i}, G_{i}^{\mathrm{SCl}} / G_{i}$, and $G_{i}^{\mathrm{XCl}} / G_{i}$ are nonlinear functions of connections' PSDs, frequencies, and channel spacings, their computations incur great complexity. To resolve this, we upperapproximate these functions with piecewise linear convex functions ${ }^{7}$, i.e., a nonlinear function $f(x)$ is fitted by a set of functions in the form $f(x) \leq \hat{f}(x)=\max \left\{a_{1}^{T} x+b_{1}, \ldots, a_{k}^{T} x+b_{k}\right\}$, so that $\hat{f}$ overestimates and approximates $f$ everywhere. The upper-approximation guarantees the resulting solutions always have feasible SNRs.

\section{MILP Formulation}

Based on the nonlinear model and its linear upper-approximation, we propose an mixed integer linear programing (MILP) to minimize the maximum bandwidth usage. The MILP takes the following input parameters: $z_{l, n} \in \mathbb{B}=\{0,1\}$, $z_{l, n}=1$ if node $n$ is on link $l ; v_{i, n} \in \mathbb{B}, v_{i, n}=1$ if node $n$ is source or destination of connection $i$; $c_{k} \in \mathbb{R}_{\geq 0}$, the spectral efficiency of modulation format $k ; \Lambda_{i} \in \mathbb{R}_{\geq 0}$, the bit rate requirement of connection $i ; \mathcal{N}$, the set of all nodes; $\mathcal{E}$, the set of all links; $\mathcal{D}$, the set of all connections; $N_{l} \in \mathbb{R}_{\geq 0}$, the length of link $l$ in number of spans.

The MILP comprises the following variables: $B_{i} \in \mathbb{R}_{\geq 0}, g_{i} \in \mathbb{R}_{\geq 0}$, and $f_{i} \in \mathbb{R}_{\geq 0}$ are the bandwidth, PSD, and the lowest frequency of spectrum 
allocated to connection $i$, respectively; $p_{i l} \in \mathbb{B}$, $p_{i l}=1$ if link $l$ is on the route of connection $i$; $q_{\text {in }} \in \mathbb{B}, q_{i n}=1$ if node $n$ is on the route of connection $i ; y_{i j} \in \mathbb{B}, y_{i j}=1$ if connection $i$ and $j$ share at least one common link; $u_{i j} \in \mathbb{B}, u_{i j}=1$ if $y_{i j}=1$ and $f_{i}+B_{i} \leq f_{j} ; w_{i j l} \in \mathbb{B}, w_{i j l}=1$ if $p_{i l}=1$ and $p_{j l}=1 ; f_{i j} \in \mathbb{R}_{\geq 0}$, the channel spacing between connection $i$ and $j$ if $y_{i j}=1$; $m_{i k} \in \mathbb{B}, m_{i k}=1$ if connection $i$ uses modulation format $k$; $t_{i j l}^{\mathrm{xci}}$, the $\mathrm{XCl}$ from connection $j$ to $i$ on link $l ; t_{i l}^{\text {sci }}$ and $t_{i l}^{\text {ase }}$, the SCI and ASE on link $l$ for connection $i$. With this notation, the optimization problem is

$$
\begin{aligned}
& \min . \quad \zeta+\varepsilon \sum_{i \in \mathcal{D}} t_{i}^{\mathrm{nli}} \\
& \text { s.t. } \\
& \sum_{l \in \mathcal{E}} p_{i l} z_{l, n}=2 q_{i n}-v_{i, n}, \\
& p_{i l}+p_{j l} \leq 1+y_{i j}, w_{i j l} \leq p_{i l}, \\
& w_{i j l} \leq p_{j l}, w_{i j l} \geq p_{i l}+p_{j l}-1, \\
& u_{i j}+y_{i j}=2 \rightarrow\left\{\begin{array}{l}
f_{i}+B_{i} \leq f_{j} \\
f_{i j}=f_{j}+\frac{B_{j}}{2}-\left(f_{i}+\frac{B_{i}}{2}\right)
\end{array}\right. \\
& u_{i j}+y_{i j}=1 \rightarrow\left\{\begin{array}{l}
f_{j}+B_{j} \leq f_{i} \\
f_{i j}=f_{i}+\frac{B_{i}}{2}-\left(f_{j}+\frac{B_{j}}{2}\right)
\end{array}\right. \\
& B_{i}=\sum_{k \in \mathcal{M}} \frac{\Lambda_{i}}{c_{k}} m_{i k}, \sum_{k \in \mathcal{M}} m_{i k}=1, \\
& w_{i j l}=1 \rightarrow t_{i j l}^{\mathrm{xci}} \geq \hat{f}^{\mathrm{xci}}, \\
& p_{i l}=1 \rightarrow\left\{\begin{array}{l}
t_{i l}^{\mathrm{sci}} \geq \hat{f}^{\mathrm{sci}} \\
t_{i l}^{\mathrm{ase}} \geq \hat{f}^{\mathrm{ase}},
\end{array}\right. \\
& t_{i}^{\mathrm{nli}}=\sum_{l \in \mathcal{E}} \sum_{j \in \mathcal{D}, j \neq i} t_{i j l}^{\mathrm{xci}}+t_{i l}^{\mathrm{sci}}+t_{i l}^{\mathrm{ase}}, \\
& t_{i}^{\mathrm{nli}}-\sum_{k \in \mathcal{M}} m_{i k} / \mathrm{SNR}_{m_{i k}}^{\mathrm{th}} \leq 0, \\
& f_{i}+B_{i} / 2 \leq \zeta,
\end{aligned}
$$

where $u \rightarrow C$ means the constraint $C$ needs to be satisfied if the condition $u$ is true, but can be dropped otherwise, and $\hat{f}{ }^{\mathrm{xci}}, \hat{f}{ }^{\mathrm{sci}}$, and $\hat{f}^{\text {ase }}$ are the linear upper-approximations of $G_{i}^{\mathrm{XCl}} / G_{i}, G_{i}^{\mathrm{SCl}} / G_{i}$, and $G_{i}^{\mathrm{ASE}} / G_{i}$, respectively. Constraint (1b) is Kirchhoff's law for flows; (1c) is the link sharing of two connections; (1d)-(1e) ensure nonoverlapping spectrum allocation; (1f) selects modulation formats and calculates connection bandwidth; (1g)-(1i) define the $\mathrm{XCI}, \mathrm{SCI}, \mathrm{ASE}$, and the total NLI of all the connections; (1j) ensures satisfactory SNR; and (1k) defines the bandwidth usage that is minimized. In the objective, the total NLI is associated with a very small weight $(\varepsilon=0.01)$ to minimize total NLI if there are more than one solutions with the minimal bandwidth. For simple networks, the solution is obtained by Gurobi Optimizer software ${ }^{8}$.

\section{Problem Decomposition}

For realistic networks, solving (1) to optimality is prohibitive due to the many integer variables and constraints. To address this, we propose a heuristic that breaks down (1) into subproblems: (i) routing and modulation level pooling (RMLP), (ii) spectrum ordering assignment (SOA), and (iii) frequency and PSD assignment (FPA) subproblems and solves them separately and sequentially.

$R M L P$ : It takes traffic requests and network topology as input, and outputs a pool of routings in the form of $p_{i l}, q_{i n}, w_{i j l}, y_{i j}, m_{i k}, B_{i}$ defined in (1). First, for each connection $i$ the $k$-shortest paths are calculated ( $k=5$ in this paper), and for each path the SNR is estimated by the LOGON method ${ }^{9}$. The two highest order modulations whose SNR requirements can be met by the path SNR estimation are selected, and the paths and their associated modulations are stored as a set of routings in $\mathcal{P}_{i}$. Note that the same path with different modulations are taken as different routings. Then we solve a low complexity MILP

$$
\begin{aligned}
& \operatorname{minimize} \quad \eta \\
& \text { s.t. } \\
& \sum_{p \in \mathcal{P}_{i}} x_{p}=1, \forall i \in \mathcal{D} \\
& F_{l}=\sum_{i \in \mathcal{D}} \sum_{\left\{p \in \mathcal{P}_{i} \mid l \in p\right\}} B_{p} x_{p} \\
& \eta \geq F_{l}, \forall l \in \mathcal{E} \\
& \eta \geq \eta_{0},
\end{aligned}
$$

where $x_{p} \in \mathbb{B}, x_{p}=1$ if routing $p$ is chosen by (2), $B_{p}$ is its number of subcarriers, $l \in p$ means that link $l$ is on routing $p, \eta$ is the estimated maximum bandwidth usage assuming wavelength converters available at each node, and (2e) is included to cut-off solutions that have been searched already. One solution to (2) represents a joint routing for all the connections. Realizing that (2) might have non-unique solutions, we try to collect as many of them as possible. Moreover, different from previous algorithms ${ }^{10}$ that solve (2) only once, we repeat it for several times. Initially, $\eta_{0}$ is set to 0 , and after the first solving, $\eta_{0}$ is set to $\eta^{*}+1$, where $\eta^{*}$ is the optimum obtained from the previous solving of (2). Finally, RMLP outputs a pool of joint routings for all the connections, from which the best in terms of (1a) is chosen in SOA and FPA.

$S O A$ : It takes the routing pool from RMLP as input, and outputs the ordering of connections in spectrum, i.e., $u_{i j}$ in (1). For each routing scheme in the pool, the connections' SNRs are first estimated by the LOGON method. Secondly, the connections are processed in an iterative way. In the $k^{\text {th }}$ iteration, the un-allocated connection with the lowest estimated SNR is found and denoted as $r_{k}$, then the largest (in bandwidth) un-allocated connection disjoint (not sharing links) with $r_{k}$ is found 


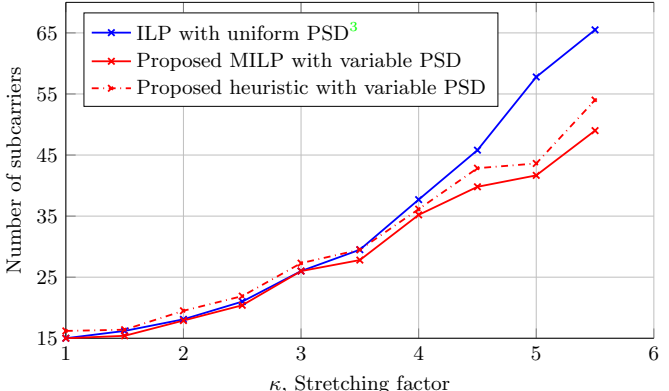

Fig. 1: Up to $28 \%$ bandwidth reduction is achieved by optimizing the transmit power of each connection separately (variable PSD). A 6-node network is considered, whose link lengths are stretched by $\kappa$. The proposed heuristic is always within $11 \%$ of the optimal solution.

and denoted as $s_{k}$. Then all the un-allocated connections disjoint with $r_{k}$ and $s_{k}$ (including $r_{k}$ and $s_{k}$ ) are grouped in set $\mathcal{Q}_{k}$. Finally, $\mathcal{Q}_{k}$ is allocated immediately after $\mathcal{Q}_{k-1}$ in spectrum, and $u_{i j}$ in (1) is thus determined. By grouping disjoint connections and allocating those with low SNR first, this procedure achieves a balance between satisfactory SNR and efficient spectrum allocation.

FPA: Taking the outputs from previous stages, FPA gives the final solution. This is done by dropping constraints (1b)-(1f) and solving (1), with $p_{i l}, q_{i n}, w_{i j l}, u_{i j}, y_{i j}, m_{i k}, B_{i}$ as input parameters. FPA is carried out for each candidate in the pool generated by RMLP, and the best one in terms of (1a) is chosen as final output.

\section{Numerical Results}

We present results for two network topologies: a simple 6-node network ${ }^{10}$ where we allow for stretching each link with a factor $\kappa \geq 1$, and the larger 14-node NSF network ${ }^{4}$. The parameters are set to $\alpha=0.22 \mathrm{~dB} / \mathrm{km}, \gamma=$ $1.32(\mathrm{~W} \cdot \mathrm{km})^{-1}, \beta_{2}=-21.7 \mathrm{ps}^{2} / \mathrm{km}, \nu=$ $193.55 \mathrm{THz}, n_{\mathrm{sp}}=1.8, L=100 \mathrm{~km}$, and the bandwidth of one subcarrier is $6.25 \mathrm{GHz}$. Each connection's $B E R$ requirement is $10^{-3}$, and its bit rate is uniformly distributed between 12 to 375 Gbps.

For the simple network, we compare the variable PSD MILP formulation with the uniform PSD ILP formulation ${ }^{3}$. To compare the two schemes for different network sizes, we set $\kappa$ from 1 to 5.5 with a step size of 0.5 . For $\kappa>5.5$, the network is too large for any connections to be feasible. The bandwidth usage as a function of the stretching factor is shown for both schemes, averaged for ten random request matrices. Fig. 1 shows that variable PSD is beneficial when the network is large, which suggests that the accumulated NLI through long paths can be mitigated by proper management of transmitter power. To verify the effectiveness of the proposed heuristic, it is plot-

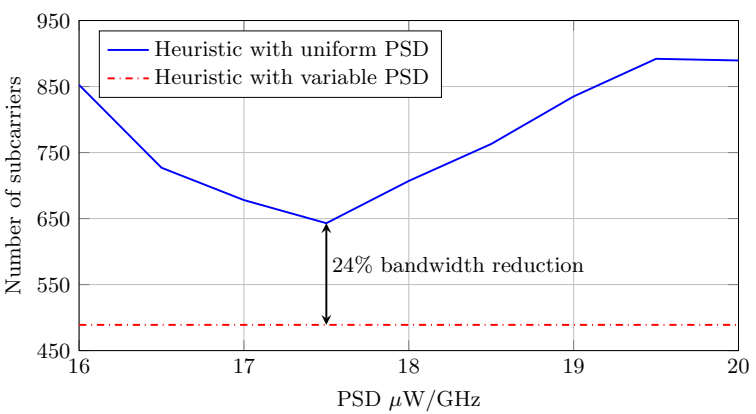

Fig. 2: Variable PSD achieves $24 \%$ bandwidth saving for 14-node NSF network compared with uniform PSD.

ted in Fig. 1. The heuristic is always within $10 \%$ of the optimal solution.

For the larger NSF network, the proposed heuristic is applied to variable and uniform PSD. Fig. 2 shows the resulting bandwidth usages, averaged for ten request matrices. The variable PSD scheme achieves $24 \%$ bandwidth reduction.

In the variable PSD scheme, most of the connections have PSDs that are exactly enough to satisfy the SNR requirements. This can reduce the overall NLI, and thus allow higher spectral efficiency modulation formats to exist, i.e., SNR margins in the uniform PSD scheme is trade for bandwidth reduction by varying PSDs individually.

\section{Conclusion}

Based on the NLI model, the benefits of varying the transmitter power for each connection in flexible-grid OOFDM networks is investigated through both MILP optimization and problem decompositions. Numerical calculations demonstrate $24 \%$ bandwidth savings for a realistic network compared with the uniform PSD scheme.

\section{References}

[1] P. Johannisson et al., "Modeling of nonlinear signal distortion in fiber-optic networks," JLT, 2014.

[2] P. Poggiolini et al., "The GN-model of fiber non-linear propagation and its applications," JLT, 2014.

[3] J. Zhao et al., "Nonlinear impairment aware resource allocation in elastic optical networks," in OFC, 2015.

[4] D. J. Ives et al., "Adapting transmitter power and modulation format to improve optical network performance utilizing the gaussian noise model of nonlinear impairments," $J L T, 2014$

[5] L. Yan et al., "Resource allocation in nonlinear flexiblegrid fiber-optic networks," in OFC, 2015.

[6] L. Yan et al., "Link-level resource allocation for flexiblegrid nonlinear fiber-optic communication systems," JLT, 2015.

[7] A. Magnani et al., "Convex piecewise-linear fitting," Optimization and Engineering, 2009.

[8] Gurobi Optimization, Inc., "Gurobi optimizer reference manual." http: //www. gurobi . com, 2015.

[9] P. Poggiolini et al., "The LOGON strategy for lowcomplexity control plane implementation in newgeneration flexible networks," in OFC, 2015.

[10] K. Christodoulopoulos et al., "Elastic bandwidth allocation in flexible OFDM-based optical networks," JLT, 2011. 